\title{
Correction to: Functional Analysis and Applications
}

\section{Correction to:}

\author{
A. H. Siddiqi, Functional Analysis and Applications, \\ Industrial and Applied Mathematics, \\ https://doi.org/10.1007/978-981-10-3725-2
}

In the original version of the book, the belated corrections from author in Chaps. 1-5, 7, 9-15, Appendix and References have been incorporated. The correction book has been updated with the changes. 